\title{
A Steady State Phase Change Problem*
}

\author{
By A. Solomon
}

In a previous paper [4] the solution to the Stefan problem for a one-dimensional semi-infinite slab with constant boundary and initial conditions was shown to be given by the limit of solutions to a nonlinear parabolic equation for the "specific internal energy." In this paper we obtain the same result for the Stefan problem in a bounded two- or three-dimensional domain, with constant boundary conditions. This result further justifies the application of the methods of [3], [4] to the Stefan problem in higher dimensions.

In Section 1 the problem to be solved is stated, and a simple solution given. In Sections 2, 3 this solution is shown to be obtainable from a limit of solutions to a related problem for the specific internal energy, as well as a solution to a related problem in the calculus of variations.

1. Notation and Statement of the Problem. Let $\&$ be a bounded region of the $x, y$ plane having a smooth boundary $\Gamma$ and consisting of material which undergoes a change of phase, from Phase "I" to Phase "II," at the critical temperature $T_{c}$ (see Fig. 1); our results apply as well for a three-dimensional region. (Phases I and II can represent "frozen" and "melted" states of the material.) Let $H$ be the latent heat of the material which is lost in the transition from Phase II to Phase I, $c_{1}, K_{1}$ and $c_{2}, K_{2}$ the specific heat and conductivity of Phase I and Phase II material, respectively, and $\kappa_{i}=K_{i} / c_{i} \rho, i=1,2$, where $\rho$ is the density of Phase I and II material, which we assume to be the same.

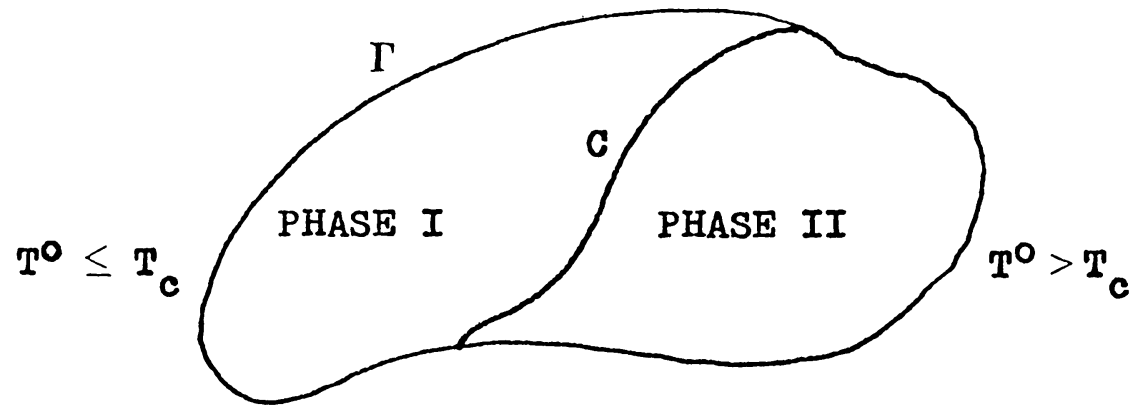

FIgURE 1. The domain $\mathfrak{L}$

Suppose that the temperature $T$ of the boundary $\Gamma$ is given by the function

$$
T(\sigma)=T^{0}(\sigma)
$$

(with $\sigma$ the arc length on $\Gamma$ ) and maintained at this temperature for all time. $T^{0}$ is to be a bounded and piecewise continuous function of $\sigma$ assuming values above and below $T_{c}$. Then the steady state temperature $T(x, y)$ at points $(x, y)$ of $\mathfrak{L}$ is har-

Received November 28, 1966.

* This paper represents results obtained at the Courant Institute of Mathematical Sciences. New York University, under grant NSF-GP-3657 with the National Science Foundation. 
monic throughout $\mathfrak{L}$ except across certain curves $C$ in $\mathscr{L}$ marking the interface between Phase I and II materials. At $C$,

$$
\begin{aligned}
T & =T_{c}, \\
K_{1}\left|\operatorname{grad} T^{-}\right| & =K_{2}\left|\operatorname{grad} T^{+}\right|,
\end{aligned}
$$

where $T^{-}, T^{+}$denote the limiting temperatures at $C$ from within the Phase $\mathrm{I}$ and Phase II regions of $\mathfrak{L}$, respectively.

We wish to determine a function $T(x, y)$ and curves $C$, which satisfy $(1 \mathrm{a}, \mathrm{b}, \mathrm{c})$ for given $T^{0}$. This can easily be done by a simple nonlinear change of variables. For let

$$
K(\beta)= \begin{cases}K_{1}, & \text { for } \beta \leq T_{c} \\ K_{2}, & \text { for } \beta>T_{c}\end{cases}
$$

and

$$
U(x, y)=\int_{0}^{T(x, y)} K(\beta) d \beta
$$

then

$$
U_{x x}+U_{y y}=0
$$

in the Phase I and II regions, while at $C|\operatorname{grad}| U^{+}|=| \operatorname{grad} U^{-} \mid$, with $U^{-}, U^{+}$the limiting values of $U$ on $C$ from within the Phase I and II regions, respectively. Thus $U$ can be considered harmonic throughout $\&$. On $\Gamma$,

$$
U(\sigma)=U^{0}(\sigma)=\int_{0}^{T^{\circ}(\sigma)} K(\beta) d \beta
$$

A harmonic function $U(x, y)$ satisfying (2c, d) exists and can be found using well-known methods of potential theory (see [1]). Since the function $K$ of (2a) never vanishes, one can solve $(2 \mathrm{~b})$ for the function $T(x, y)$ in terms of $U(x, y)$, which obeys (1a, b, c); moreover the interface curve $C$ on which $T=T_{c}$ is simply the equipotential curve for $U$ on which

$$
U=\int_{0}^{T_{c}} K(\beta) d \beta .
$$

2. A Related Problem for "Energy." Define $T$ and a function $\kappa$ as functions of a new variable $e$ by

$$
\begin{gathered}
T(e)=\left\{\begin{array}{l}
T_{c}+(e-H) / c_{1}, \text { for } e<H ; \\
T_{c}, \text { for } H \leq e \leq 2 H ; \\
T_{c}+(e-2 H) / c_{2}, \text { for } e>2 H
\end{array}\right. \\
\kappa(e)=\left\{\begin{array}{l}
\kappa_{1}, \text { for } e \leq H ; \\
\phi_{1}(e), \text { for } H \leq e \leq H+\epsilon ; \\
\delta, \text { for } H+\epsilon \leq e \leq 2 H-\epsilon ; \\
\phi_{2}(e), \text { for } 2 H-\epsilon \leq e \leq 2 H \\
\kappa_{2}, \text { for } 2 H \leq e
\end{array}\right.
\end{gathered}
$$


where $\epsilon, \delta$ are any (small) positive numbers, and $\phi_{1}, \phi_{2}$ are any smooth monotonic functions such that $\kappa(e), \kappa^{\prime}(e)$ are continuous. Let $E^{0}(\sigma)$ be defined on $\Gamma$ in such a way that $T\left(E^{0}(\sigma)\right)=T^{0}(\sigma)$ (by (3)). Consider the boundary value problem

$$
\begin{aligned}
\left(\kappa(e) e_{x}\right)_{x}+\left(\kappa(e) e_{y}\right)_{y} & =0 \text { on } \mathscr{L} ; \\
e & =E^{0} \text { on } \Gamma .
\end{aligned}
$$

We claim that an analytic solution of $(5 \mathrm{a}, \mathrm{b})$ exists, which as $\epsilon, \delta$ tend to zero, converges to a function yielding by (3) a piecewise harmonic function $T$ obeying $(1 \mathrm{a}, \mathrm{b}, \mathrm{c})$.

Let

$$
F(e)=\int_{0}^{e} \kappa(\beta) d \beta ;
$$

then as a function of $x, y, F$ obeys (by $(5 \mathrm{a}, \mathrm{b}))$

$$
\begin{aligned}
F_{x x}+F_{y y} & =0 \text { on } \mathscr{L}, \\
F(\sigma) & =\int_{0}^{E^{\circ}(\sigma)} \kappa(\beta) d \beta \text { on } \Gamma .
\end{aligned}
$$

Under the assumptions on $E^{0}, \kappa$, such a function $F$ exists, and since $F^{\prime}(e)=\kappa(e) \neq 0$, $e$ and $T$ may be found by (6), (3).

The equipotential curves for $F$ on which $e, F$ are constant, are Jordan arcs joining points of $\Gamma$. Let $\mathfrak{L}^{-}, \mathfrak{L}^{0}, \mathfrak{L}^{+}$be the subsets of $\mathfrak{L}$ in which $e<H, H<e<2 H$, $e>2 H$, respectively. These regions are bounded by smooth Jordan arcs or sets of curves $C^{H}, C^{2 H}$ in $\&$ on which $e=H, F=\kappa_{1} H$, and $e=2 H, F=\kappa_{1} H+\int_{H}^{2 H} \kappa(\beta) d \beta$, respectively. The regions clearly depend on $\epsilon, \delta$.

Let $\epsilon$ tend to zero, with $\kappa(e)$ converging in a decreasing manner to the piecewise constant function

$$
\kappa(e)=\left\{\begin{array}{l}
\kappa_{1}, \quad \text { for } e \leq H ; \\
\delta, \text { for } H<e<2 H ; \\
\kappa_{2}, \text { for } e \geq 2 H ;
\end{array}\right.
$$

by Dini's theorem $F$ converges uniformly on $\Gamma$ to a continuous function given by (6b) (see [2, p. 106]). Consequently, as $\epsilon$ tends to $0, F$ converges uniformly on $\mathscr{L}+$ $\Gamma$ to a harmonic function satisfying $(6 \mathrm{a}, \mathrm{b})$ with $\kappa$ defined by $(7)$. By Harnack's theorem the domains $\mathfrak{L}^{+}, \mathfrak{L}^{0}, \mathfrak{L}^{-}$and curves $C^{H}, C^{2 H}$ converge to domains $\mathfrak{L}^{+}, \mathfrak{L}^{0}, \mathfrak{L}^{-}$ and smooth Jordan $\operatorname{arcs} C^{H}, C^{2 H}$ as above. From (6),

$$
\begin{aligned}
F & =\kappa_{1} e \leq \kappa_{1} H \quad \text { on } \quad \mathcal{L}^{-} ; \\
\kappa_{1} H & \leq F=\kappa_{1} H+\delta(e-H) \leq H\left(\kappa_{1}+\delta\right) \text { on } \mathfrak{L}^{0} ; \\
H\left(\kappa_{1}+\delta\right) & \leq F=H\left(\kappa_{1}+\delta\right)+\kappa_{2}(e-2 H) \text { on } \mathfrak{L}^{+},
\end{aligned}
$$

and

$$
F=\left\{\begin{array}{l}
\kappa_{1} H \text { on } C^{H} ; \\
H\left(\kappa_{1}+\delta\right) \text { on } C^{2 H}
\end{array}\right.
$$

From (6c), 


$$
|\operatorname{grad} F|=\left\{\begin{array}{l}
\kappa_{1}|\operatorname{grad} e|, \text { in } \mathfrak{L}^{-} ; \\
\left(\kappa_{1}+\delta\right)|\operatorname{grad} e|, \text { in } \mathfrak{L}^{0}, \\
\kappa_{2}|\operatorname{grad} e|, \text { in } \mathfrak{L}^{+} .
\end{array}\right.
$$

Since $F(x, y)$ is constant on $C^{H}$ it increases as the point $(x, y)$ crosses $C^{H}$ from $\mathfrak{L}^{-}$ to $\mathfrak{L}^{0}$. Let $e^{-}, e^{0}$ be the limiting values of $e$ on $C^{H}$ from within $\mathscr{L}^{-}$and $\mathfrak{L}^{0}$ respectively.

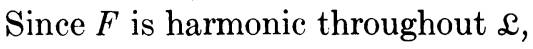

$$
\left|\operatorname{grad} e^{0}\right|\left(\kappa_{1}+\delta\right)=\kappa_{1} \mid \operatorname{grad} e^{-1}
$$

on $C^{H}$ (Eq. 8). Similarly, letting $e^{+}, e^{0}$ be the limiting values of $e$ on $C^{H}, C^{2 H}$, from $\mathfrak{L}^{+}, \mathfrak{L}^{0}$ respectively,

$$
\left|\operatorname{grad} e^{+}\right| \kappa_{2}=\left(\kappa_{1}+\delta\right)\left|\operatorname{grad} e^{0}\right| ;
$$

thus by $(6), e$ is harmonic within $\mathfrak{L}^{0}, \mathfrak{L}^{-}, \mathfrak{L}^{+}$, continuous throughout $\mathfrak{L}$, and has a discontinuous gradient across $C^{H}, C^{2 H}$.

Let $\delta$ tend to 0 . By reasoning similar to that above, $\kappa(e)$ converges to the step function

$$
\kappa(e)= \begin{cases}\kappa_{1}, & \text { for } e \leq H ; \\ 0, & \text { for } H<e<2 H ; \\ \kappa_{2}, & \text { for } e \geq 2 H,\end{cases}
$$

and $F$ converges uniformly on $\mathfrak{L}^{+}$and $\Gamma$ to a harmonic function obeying $(6 \mathrm{a}, \mathrm{b})$. Moreover by (5d), $C^{H}, C^{2 H}$ converge to a common curve $C$, on which $F=H \kappa_{1}$, while $\mathfrak{L}^{0}$ tends to the empty set, and $\mathfrak{L}^{+}, \mathfrak{L}^{-}$tend to sets $\mathfrak{L}^{\mathrm{II}}, \mathfrak{L}^{\mathrm{I}}$, on which $F>\kappa_{1} H$ and $F<\kappa_{1} H$ respectively. The functions $e$ converge to a function harmonic on $\mathfrak{L}^{\mathrm{II}}$ and $\mathfrak{L}^{\mathrm{I}}$, and

$$
F=\left\{\begin{array}{l}
\kappa_{1} e, \text { on } \mathscr{L}^{\mathrm{I}} ; \\
\kappa_{1} H+\kappa_{2}(e-2 H), \text { on } \mathfrak{L}^{\mathrm{II}} .
\end{array}\right.
$$

For $e^{+}, e^{-}$the limiting values of $e$ at $C$ from within $\mathfrak{L}^{\mathrm{II}}, \mathfrak{L}^{\mathrm{I}}$, respectively,

$$
e^{-}=H, e^{+}=2 H, \text { on } C \text {. }
$$

Moreover, since $\operatorname{grad} F$ is continuous over $\mathfrak{L}$,

$$
\kappa_{1}\left|\operatorname{grad} e^{-}\right|=\kappa_{2}\left|\operatorname{grad} e^{+}\right|, \quad \text { on } C
$$

and $e=E^{0}$ on $\Gamma$. Using (3), we now obtain a function $T(x, y)$ which is harmonic on $\mathfrak{L}^{\mathrm{I}}, \mathfrak{L}^{\mathrm{II}}$ and obeys $(1 \mathrm{a}, \mathrm{b}, \mathrm{c}) . T$ is the solution to the steady state problem.

Since in the limit for $\epsilon=\delta=0, F$ (and thus $T$ ) is determined uniquely by the given boundary values of $T$ on $\Gamma, T$ is uniquely determined.

3. A Related Variational Problem. As a harmonic function continuous on $\Gamma, F$ is under suitable conditions the solution to the problem of minimizing the Dirichlet integral over $\mathfrak{L}$ among all functions obeying (6b) (see [1]). This implies by (3), (6), that the solution $T$ to $(1 \mathrm{a}, \mathrm{b}, \mathrm{c})$ is that function minimizing the integral

$$
I=\frac{1}{\rho^{2}} \iint_{\mathscr{L}}(K(T))^{2}\left(T_{x}^{2}+T_{y}^{2}\right) d x d y
$$


with $K(T)$ defined by (2a), among all piecewise smooth functions $T$ obeying (1a).

Courant Institute of Mathematical Sciences

New York University

New York, New York 10012

1. R. Courant, Dirichlet's Principle, Conformal Mapping and Minimal Surfaces, Interscience, New York, 1950. MR 12, 90.

2. R. Courant, Differential and Integral Calculus, Vol. II, Interscience, New York, 1964.

3. M. Rose, "A method for calculating solutions of parabolic equations with a free boundary," Math. Comp., v. 14, 1960, pp. 249-256. MR $22 \# 6085$. 360 .

4. A. Solomon, "Some remarks on the Stefan problem," Math. Comp., v. 20, 1966, pp. 347- 